\title{
Atmospheric Transports, the Thermohaline Circulation, and Flux Adjustments in a Simple Coupled Model
}

\author{
JOCHEM MAROTZKe AND PETER H. STONE \\ Center,for Global Change Science, Department of Earth, Atmospheric, and Planetary Sciences, Massachusetts Institute of Technology. \\ Cambridge, Massachusetts
}

(Manuscript received 29 March 1994, in final form 18 October 1994)

\begin{abstract}
A theoretical analysis of the interactions between atmospheric meridional transports and the thermohaline circulation is presented, using a four-box ocean-atmosphere model in one hemisphere. The model is a simplified version of that developed by Nakamura, Stone, and Marotzke and is amenable to analytical solutions. The ocean model is Stommel's; the atmospheric model gives the surface heat and freshwater fluxes as residuals of the atmospheric energy and moisture budgets, assumed in balance. Radiation at the top of the atmosphere depends linearly on surface temperature; atmospheric meridional heat and moisture transports are proportional to the meridional temperature gradient.

A Newtonian cooling law is derived for differential surface heat flux. The restoring coefficient is proportional to the efficiency of atmospheric transports and inversely proportional to the relative ocean area compared to total surface area. Surface freshwater flux increases with increasing temperature gradient and is inversely proportional to the ratio of ocean area to catchment area. The range of stable solutions with high-latitude sinking is smaller than in related, uncoupled box models due to the dependence of freshwater flux on the temperature gradient, which leads to a positive feedback with the thermohaline circulation. A strong control of the temperature gradient by atmospheric transports enhances the positive feedback between the salinity gradient and thermohaline circulation; simultaneously, it weakens the positive feedback between atmospheric moisture transport and the thermohaline circulation.

Overestimating the atmospheric moisture transport and underestimating oceanic mass transport both artificially destabilize the high-latitude sinking state. Overestimating the atmospheric heat transport and hence the Newtonian restoring coefficient can be artificially stabilizing or destabilizing. These erroneous sensitivities are not alleviated if flux adjustments are added to obtain the correct mean climate, and then held fixed in climate change experiments. We derive alternate flux adjustment schemes, which do preserve the model's stability properties for particular sources of error.
\end{abstract}

\section{Introduction}

We present here what we believe is the simplest model of some of the large-scale ocean-atmosphere interactions that are most important for climate and climate stability. Focus is on the stability of the thermohaline overturning circulation in the North Atlantic, which contributes strongly to the total poleward heat transport in the Northern Hemisphere (Hall and Bryden 1982; Roemmich and Wunsch 1985), and which is a key manifestation of the ocean's role in climate.

Results from general circulation models show that an oceanic state without thermohaline circulation in the North Atlantic is dynamically consistent (e.g., Bryan 1986; Manabe and Stouffer 1988; Marotzke and Willebrand 1991; see Weaver and Hughes 1992, for a review). It is not clear, however, how easily the

Corresponding author address: Dr. Jochem Marotzke, Room 541514, Department of Earth, Atmospheric, and Planetary Sciences, Massachusetts Institute of Technology, Cambridge, MA 02139-4307. E-mail: jochemosound.mit.edu current global circulation pattern could be changed drastically. Uncoupled ocean general circulation models (GCMs) traditionally have mimicked airsea interactions by assuming that the atmosphere strongly controls sea surface temperature (SST) and the surface freshwater flux; both distributions are therefore effectively prescribed as external parameters (mixed thermohaline boundary conditions). Using this type of model, it was concluded that an equilibrium forming deep water at high latitudes is very sensitive to variations in freshwater flux and surface salinity (Marotzke 1990; Marotzke and Willebrand 1991; Weaver et al. 1991, 1993). Recent results (Zhang et al. 1993; Rahmstorf and Willebrand 1995 ) suggest that this sensitivity may well have been overestimated by assuming too strong control of SST by the atmosphere. However, Nakamura et al. (1994) have shown, with a five-box model of the oceanatmosphere system in one hemisphere, that another powerful positive feedback enters if the surface freshwater flux is determined interactively and not prescribed as under mixed boundary conditions. 


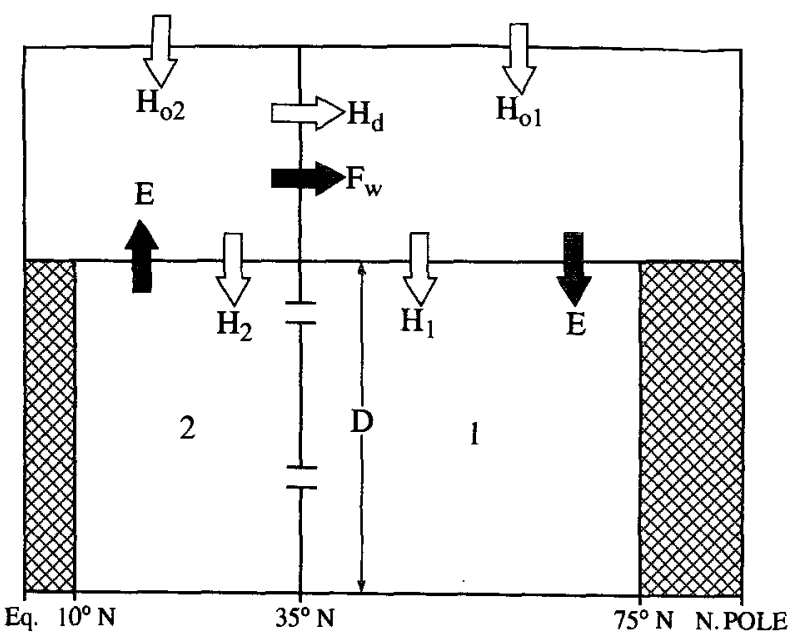

FiG. 1. Vertical cross section of the model. (See text for definitions.)

Willebrand (1993) and Marotzke (1994) have discussed some of the feedbacks that must be included to represent accurately the stability of the thermohaline circulation, but not even all the known, important feedbacks have been implemented in models capable of representing the three-dimensional structure of the thermohaline circulation. Recent results from the coupled climate model of the Geophysical Fluid Dynamics Laboratory (GFDL; Manabe and Stouffer 1993, 1994) strongly suggest that the uncoupled ocean models using mixed boundary conditions indeed overestimate the sensitivity of the thermohaline circulation. Their model, however, must use artificial heat and freshwater fluxes at the air-sea interface to reproduce the current climate (flux adjustments), and it has been shown by Nakamura et al. (1994) that models in need of flux adjustments are unlikely to have the correct stability behavior.

This paper, conceptually, takes as its starting point the work of Nakamura et al. (1994, NSM hereinafter). We have simplified their representations of the atmospheric transports to the degree that an analytical treatment is possible. The atmospheric budgets for heat and moisture are assumed linear; the only nonlinearity left in the model is the interaction between the oceanic flow strength and density gradients. The simplicity of the model allows the analysis and compact representation of a rich variety of phenomena. The paper is organized as follows. Section 2 gives the model formulation. Sections 3 and 4 discuss the resulting surface heat and freshwater fluxes, respectively. Section 5 describes the equilibrium solutions, and section 6 a linear stability analysis of the steady state with high-latitude sinking. Section 7 analyzes the effects of flux adjustments on the time-dependent model behavior; alternate adjustment schemes are derived for various sources of error, which recover the correct transient behavior. Section 8 gives a brief summary and discussion.

\section{Basic equations}

The model consists of two ocean boxes and two atmospheric boxes. The ocean boxes are well mixed and have depth $D$; box 1 represents the high-latitude ocean and box 2 the low-latitude ocean (Fig. 1). $H_{1}$ and $H_{2}$ are ocean heat gain through the surface, and $H_{01}$ and $H_{02}$ are atmospheric energy gain at the top; $H_{d}$ is the meridional energy transport in the atmosphere, $E$ is net evaporation at low latitudes and net precipitation at high latitudes, and $F_{w}$ is the meridional atmospheric moisture transport.

The conservation equations for the ocean are (cf., Stommel 1961; Marotzke 1990)

$$
\begin{aligned}
& \dot{T}_{1}=H_{1}+|q|\left(T_{2}-T_{1}\right) \\
& \dot{T}_{2}=H_{2}-|q|\left(T_{2}-T_{1}\right) \\
& \dot{S}_{1}=-H_{S}+|q|\left(S_{2}-S_{1}\right) \\
& \dot{S_{2}}=H_{S}-|q|\left(S_{2}-S_{1}\right) .
\end{aligned}
$$

The flow strength $q$ is related to the meridional density gradient by a linear law,

$$
q=k\left[\alpha\left(T_{2}-T_{1}\right)-\beta\left(S_{2}-S_{1}\right)\right],
$$

where a linear equation of state has been assumed and $\alpha$ and $\beta$ are the thermal and haline expansion coefficients. Some support for the simple relationship (5) derives from the recent results of Hughes and Weaver (1994). In (3) and (4) $H_{S}$, the virtual surface salinity flux, is related to the surface freshwater flux $E$ through

$$
H_{S}=S_{0} \frac{E}{D},
$$

where $S_{0}$ is a constant reference salinity and $E$ is water loss (gain) of the low (high) latitude ocean, measured in meters per second. Notice that in order to convert $H_{1}$ and $H_{2}$ (which have units ${ }^{\circ} \mathrm{C} \mathrm{s}^{-1}$ ) into physical heat fluxes, denoted $\tilde{H}_{1}$ and $\tilde{H}_{2}$, they are multiplied by the heat capacity of a unit water column, which is

$$
\begin{aligned}
c \rho_{0} D & \approx 4 \times 10^{6} \mathrm{~J} \mathrm{~m}^{-3} \mathrm{~K}^{-1} \times 5 \times 10^{3} \mathrm{~m} \\
& \approx 2 \times 10^{10} \mathrm{~J} \mathrm{~m}^{-2} \mathrm{~K}^{-1} .
\end{aligned}
$$

We will use the term surface heat flux interchangeably for the heat fluxes proper and for the induced temperature tendencies.

The novel features of the model used here arise from the specification of the surface heat and freshwater fluxes. In contrast to Stommel (1961), Marotzke (1990), and Huang et al. (1992) who used either restoring laws for the surface fluxes (with restoring timescale for temperature much shorter than for salinity) or prescribed temperature and surface freshwater flux, we employ here an explicit atmospheric model. 
The fundamental assumption made is that heat and moisture capacities of the atmosphere are negligible. This is justified if only timescales longer than the atmosphere's equilibration time (order 1 month) are considered. If it is then possible to specify the energy fluxes at the top of the atmosphere and the meridional energy and water transports in the atmosphere, the airsea exchanges can be determined as the residual of the steady-state atmospheric heat and moisture budgets. One avoids the explicit computation of air-sea fluxes from bulk parameterizations and, in particular, of rainfall as a function of the model state, which is near impossible in simple models and very problematic even in GCMs. The approach advocated here has been successfully taken by NSM in a simple numerical climate model; it is further simplified here to make the model amenable to analytical solutions.

Meridional atmospheric transports in middle and high latitudes are mainly accomplished by transient eddies (traveling highs and lows). NSM use a vertically integrated parameterization of meridional eddy heat and moisture fluxes based on that developed by Stone and Yao (1990), which in turn derives from baroclinic stability theory. Furthermore, NSM assume a fixed atmospheric lapse rate, with atmospheric surface temperature identical to sea surface temperature. The meridional eddy moisture fluxes in the NSM model depend on the temperature at the boundary between the atmospheric boxes, through the Clausius-Clapeyron equation, and on roughly the third power of the meridional temperature gradient. Meridional sensible heat transport likewise goes like roughly the third power of the meridional temperature gradient. These parameterizations, which find support in scaling arguments for baroclinic instability (Held 1978) and empirical studies of seasonal changes (Stone and Miller 1980), are drastically simplified here, by assuming

$$
\begin{aligned}
H_{d} & =\tilde{\chi}\left(T_{2}-T_{1}\right) \\
F_{w^{\prime}} & =\tilde{\gamma}\left(T_{2}-T_{1}\right),
\end{aligned}
$$

where $H_{d}$ and $F_{k}$ are, respectively, the meridional fluxes of heat and moisture in the atmosphere, integrated over the $35^{\circ} \mathrm{N}$ latitude circle; the tilde over $\chi$ and $\gamma$ is used for later convenience. The heat transport formulation [Eq. (8)] is physically equivalent to the two-mode approximation in North's (1975) energy balance model. Equations (8) and (9) eliminate the effect of the temperature dependence of the saturation water vapor pressure, but capture the most salient feature of Stone and Yao's (1990) parameterization: Eddy activity goes up as the meridional temperature gradient increases. Crude though the assumptions may be, they allow investigation of the effects of computing atmospheric and oceanic transports and fluxes fully interactively. NSM's numerical results will guide us in choosing sensible values for the parameters in the linear laws.
Notice that Eqs. (8) and (9) do not constitute a linearization of the dependence of the meridional transports on the meridional temperature gradient in the NSM model. NSM found approximately a third-power dependence; it is readily shown that in a linearization of a third-power law, the coefficients of the linear terms would be three times the coefficients of the linear laws, (8) and (9), given the same reference temperature gradient and transport. We will use the simpler forms (8) and (9), but realize that if they are fitted to the NSM results, the coefficients are underestimated by a factor of 3 . Constant terms in the transport specifications, whether stemming from a linearization of eddy transport formulations or contributions independent of the temperature gradient, would not cause any significant changes in model behavior and are therefore not included in (8) and (9).

The last ingredient to our simple model is the parameterization of radiation at the top of the atmosphere; following Wang and Stone (1980) and NSM, it is linearized and given as

$$
\begin{aligned}
& H_{01}=A_{1}-B T_{1} \\
& H_{02}=A_{2}-B T_{2},
\end{aligned}
$$

where $A_{1}$ and $A_{2}$ are net incoming radiation at high and low latitudes, respectively, for a surface temperature of $0^{\circ} \mathrm{C} ; A_{1}$ is negative, and $A_{2}$ is positive; $B T_{1}$ and $B T_{2}$ mark longwave fluxes at high and low latitudes, respectively, caused by deviations of surface temperature from zero.

Despite its simplicity, the system (1)-(10), together with the assumption of an equilibrated atmosphere, allows the deduction of a rich variety of phenomena, which we will now explore.

\section{Surface heat fluxes}

The heat budget for the high-latitude atmospheric box reads

$$
\int_{\text {total }} H_{01} d a-\int_{\text {ocean }} \tilde{H}_{1} d a+H_{d}=0 .
$$

The first integral is the (negative) energy gain at the top of the atmosphere, integrated over the entire area $F_{01}$ north of the latitude circle dividing the boxes. The second integral is the (likewise negative) heat loss to the ocean, integrated over the ocean portion, $F_{1}$, of the high-latitude box. The third integral is the (integrated) meridional energy flux and given by Eq. (8). It is now assumed that $H_{01}$ and $H_{1}$ are spatially constant (or, rather, that only the area-averaged values matter), and we obtain from (11)

$$
F_{01} H_{01}-F_{1} \tilde{H}_{1}+H_{d}=0
$$

and hence

$$
\tilde{H}_{1}=\frac{1}{\epsilon} H_{01}+\frac{1}{\epsilon F_{01}} H_{d}
$$


where $\epsilon \equiv F_{1} / F_{01}$, the relative ocean coverage of the high-latitude area. Analogous considerations hold for the low-latitude boxes, and we assume, for simplicity, that $F_{2}=F_{1}$ and $F_{02}=F_{01}$. Hence,

$$
\tilde{H}_{2}=\frac{1}{\epsilon} H_{02}-\frac{1}{\epsilon F_{01}} H_{d} \text {. }
$$

Using the parameterizations (8) for $H_{d}$ and (10) for $H_{01}$ and $H_{02}$, one finally arrives at expressions for the surface heat fluxes that are functions of the oceanic temperatures only:

$$
\begin{aligned}
& \tilde{H}_{1}=\frac{1}{\epsilon}\left(A_{1}-B T_{1}\right)+\frac{\tilde{\chi}}{\epsilon F_{01}}\left(T_{2}-T_{1}\right) \\
& \tilde{H}_{2}=\frac{1}{\epsilon}\left(A_{2}-B T_{2}\right)-\frac{\tilde{\chi}}{\epsilon F_{01}}\left(T_{2}-T_{1}\right) .
\end{aligned}
$$

The spatially averaged ocean heat uptake is, from (13),

$$
\tilde{H}_{1}+\tilde{H}_{2}=\frac{1}{\epsilon}\left(H_{01}+H_{02}\right) \text {. }
$$

Total ocean heat gain is independent of the meridional atmospheric transports, which only affect the spatial distribution but not the overall amount of air-sea heat exchange. Adding the oceanic heat budgets, Eqs. (1) and (2), one obtains, using (15),

$$
\dot{T}_{1}+\dot{T}_{2}=H_{1}+H_{2}=\frac{1}{\epsilon} \frac{H_{01}+H_{02}}{c \rho_{0} D} .
$$

The parameterizations for $H_{01}$ and $H_{02},(10)$, give

$$
\frac{1}{2}\left(\dot{T}_{1}+\dot{T}_{2}\right)=\frac{1}{\epsilon} \frac{A_{1}+A_{2}}{2 c \rho_{0} D}-\frac{1}{\epsilon} \frac{B}{c \rho_{0} D} \frac{\left(T_{1}+T_{2}\right)}{2} .
$$

The "global" mean temperature is restored to its equilibrium value, $\left(A_{1}+A_{2}\right) / 2 B$, with a time constant of $\epsilon \mathcal{C} \rho_{0} D / B$. Reasonable values are $A_{1} \approx-40 \mathrm{~W} \mathrm{~m}^{-2}$, $A_{2} \approx 90 \mathrm{~W} \mathrm{~m}^{-2}$, and $B \approx 1.7 \mathrm{~W} \mathrm{~m}^{-2} \mathrm{~K}^{-1}$ (e.g., NSM), giving a steady-state mean temperature of some $15^{\circ} \mathrm{C}$ and a restoring timescale of about $\epsilon 10^{10} \mathrm{~s}$, or roughly 150 years if the hemisphere under consideration is half covered by ocean.

The more interesting quantity is the meridional temperature difference

$$
T \equiv T_{2}-T_{1}
$$

for which we obtain, from oceanic heat conservation, Eqs. (1) and (2), and the parameterizations for the surface fluxes, Eq. (14),

$$
\begin{aligned}
\dot{T} & =H_{2}-H_{1}-2|q| T \\
& =\frac{1}{\epsilon}\left(\frac{A_{2}-A_{1}}{c \rho_{0} D}\right)-\frac{1}{\epsilon}\left(\frac{2 \chi+B}{c \rho_{0} D}\right) T-2|q| T,
\end{aligned}
$$

where $\chi \equiv \tilde{\chi} F_{01}^{-1}$. Assume, first, that neither atmosphere nor ocean transport heat; that is, that both $\chi$ and $q$ are zero. The steady-state meridional temperature gradient would then be determined by the radiation balance alone, and given as

$$
\left.T_{R} \equiv T\right|_{\chi=0, q=0}=\frac{A_{2}-A_{1}}{B} .
$$

For the parameters adopted here, $T_{R}$ is about $76^{\circ} \mathrm{C}$. Next, assume that only the atmosphere transports heat horizontally, but not the ocean. The steady-state temperature, thus defined, would be the equilibrium temperature $T_{E}$ introduced by Bretherton (1982). It is derived from a balance purely between dynamical and radiative transports in the atmosphere; the ocean's role in heat transport is neglected. From (19), $T_{E}$ is readily found as

$$
\left.T_{E} \equiv T\right|_{q=0}=\frac{A_{2}-A_{1}}{2 \chi+B} .
$$

To evaluate (21), we have to assume values for $\tilde{\chi}$ and $F_{01}$. In their standard run, NSM obtain $T=29^{\circ} \mathrm{C}$ and $H_{d}=4.7 \mathrm{PW}\left(1 \mathrm{PW} \equiv 10^{15} \mathrm{~W}\right)$, which, together with $F_{01} \approx 1.25 \times 10^{14} \mathrm{~m}^{2}$, gives $\chi \approx 1.3 \mathrm{~W} \mathrm{~m}^{-2} \mathrm{~K}^{-1}$. Hence, $T_{E}$ is about $30^{\circ} \mathrm{C}$; atmospheric transports reduce the purely radiative temperature contrast by more than one half.

From (19), the surface heat fluxes driving the meridional temperature gradient can be rewritten:

$$
H_{2}-H_{1}=\lambda\left(T_{E}-T\right),
$$

with

$$
\lambda \equiv-\left(\frac{\partial\left(H_{2}-H_{1}\right)}{\partial T}\right)=\frac{1}{\epsilon}\left(\frac{2 \chi+B}{c \rho_{0} D}\right)
$$

and $T_{E}$ given by (21), and we obtain

$$
\dot{T}=\lambda\left(T_{E}-T\right)-2|q| T .
$$

Equation (22) is a Newtonian cooling law for ocean temperature, which has been widely used as a boundary condition on SST in numerical ocean models (e.g., Haney 1971; Marotzke and Willebrand 1991). Notice, however, that (22) connects spatial differences in surface heat flux to temperature differences. Equation (17) shows that the spatial-mean heat flux can likewise be written as a Newtonian law for the mean temperature, but spatial mean and difference are restored with different time constants. This "scale dependence" of the restoring coefficient is equivalent to the heat fluxes $H_{1}$ and $\mathrm{H}_{2}$ not being computable by a purely local relationship, but involving horizontal atmospheric transports [see Eq. (14)]. Bretherton (1982), Willebrand (1993), and Marotzke (1994) have discussed the relationship between atmospheric transports and a restoring boundary condition for model SST, but the present model shows the connection in its purest form, as will be shown now.

Without oceanic heat transport (equivalent here to zero flow, $q=0), T=T_{E}$ in steady state, and the 
surface heat flux is zero. But if there are currents in the ocean, they tend to reduce $T$, which is counteracted by the surface heat fluxes trying to maintain $T=T_{E}$. The steady state of Eq. (24) obeys the relation (note that $q$ depends on $T$ and $S$ )

$$
T=\left(1+\frac{2|q(T, S)|}{\lambda}\right)^{-1} T_{E}
$$

so the amount by which $T$ differs from $T_{E}$ depends on the relative sizes of the timescales $q^{-1}$ and $\lambda^{-1}$. With a meridional volume transport of $10 \mathrm{~Sv}\left(\mathrm{~Sv} \equiv 10^{6}\right.$ $\mathrm{m}^{3} \mathrm{~s}^{-1}$ ) and a volume of the high-latitude basin of $10^{17}$ $\mathrm{m}^{3}(5000 \mathrm{~km} \times 4000 \mathrm{~km} \times 5000 \mathrm{~m})$, the advective (flushing) timescale $q^{-1}$ is $10^{10} \mathrm{~s}$ or 300 years. Multiplication of $q$ by the heat capacity of a unit water column, $2 \times 10^{10} \mathrm{~J} \mathrm{~m}^{-2} \mathrm{~K}^{-1}$, gives a heat flux per unit column of the thermohaline circulation of $2 \mathrm{~W} \mathrm{~m}^{-2}$ $\mathrm{K}^{-1}$, a little larger than the coefficient of longwave radiation $B$.

The surface heat flux per degree deviation of $T$ from $T_{E}$, from the Newtonian cooling law, Eqs. (22) and (23), is $\epsilon^{-1} \times 4.3 \mathrm{~W} \mathrm{~m}^{-2} \mathrm{~K}^{-1}$, meaning that for $\epsilon$ as large as $1 / 2, \lambda$ is four times greater than $q$. If one assumes that the ocean's heat transport is solely accomplished by its thermohaline circulation, which is confined to the Atlantic in the Northern Hemisphere, the appropriate $\epsilon$ is $1 / 6$, making the timescale of the Newtonian cooling about 25 years, an order of magnitude smaller than the advective timescale. From Eq. (25), the expected meridional temperature gradient is thus about $25^{\circ} \mathrm{C}$, as opposed to $30^{\circ} \mathrm{C}$ without oceanic heat transport. But we see that oceanic temperature is very strongly controlled by the atmosphere, essentially because radiative and dynamical atmospheric transports act to quickly reduce deviations in meridional temperature gradient from $T_{E}$. This effect is amplified because the ocean does not cover the entire globe $(\epsilon<1)$. Assume that $T$ is less than its steady-state value, for example, because there is a positive temperature anomaly at high latitudes. Upward longwave radiative flux will increase at high latitudes, and atmospheric meridional transport will decrease. To balance the atmospheric heat budget, the ocean must give off more heat to the atmosphere but it has an area of a factor $\epsilon$ less, so per unit area the oceanic heat loss is very large, and the temperature anomaly is rapidly wiped out.

If the atmospheric heat transport depends on $T$ with a power $n$ greater than unity, the effective restoring coefficient from a linearization is $n$ times the $\lambda$ used here ( see section 2 ). In particular, $n=3$ as appropriate in NSM would yield a restoring coefficient of almost $80 \mathrm{~W} \mathrm{~m}^{-2} \mathrm{~K}^{-1}$, for $\epsilon=1 / 6$. Consistent with the findings of NSM, this corresponds to a restoring timescale of about 8 years for a layer $5000 \mathrm{~m}$ deep, or 1 month for a layer $50 \mathrm{~m}$ deep, which is the value commonly used in ocean GCMs. We find, somewhat surprisingly, that this value is not necessarily too large but corresponds to the limit of a small ocean basin and relatively strong dependence of atmospheric heat transport on temperature gradient.

Anomalies of the mean temperature, $T_{1}+T_{2}$, are eliminated on a timescale that is given by $\epsilon / B$ only [Eq. (17)]; the process is less efficient because dynamical atmospheric transports do not enter. Bretherton (1982) argued that the smaller-scale SST anomalies are, the more efficient are the induced anomalous atmospheric transports and thus the removal of the original anomaly. A one-dimensional example demonstrating this effect has been presented by Marotzke (1994). While the timescales of the removal of anomalies of the mean and anomalies of the meridional gradient differ by only a factor of 2 in the present model, we clearly identify the causal connection between efficiency of atmospheric transports and the Newtonian cooling law. In particular, atmospheric heat capacity does not enter the discussion in any way since the atmosphere is assumed in steady state (the storage terms are neglected ).

\section{Surface freshwater fluxes}

With their standard parameterization, NSM obtain a meridional atmospheric moisture flux $F_{w}$ of $0.44 \mathrm{~Sv}$ across the entire latitude circle of $35^{\circ} \mathrm{N}$, induced by a temperature contrast of $29^{\circ} \mathrm{C}$. If obtained from the linear relationship (9), these values would correspond to a coefficient $\tilde{\gamma}$ of $1.5 \times 10^{4} \mathrm{~m}^{3} \mathrm{~s}^{-1} \mathrm{~K}^{-1}$. Then $F_{w}$ must be balanced by net evaporation at low latitudes and net precipitation at high latitudes. In analogy to the high-latitude atmospheric heat budget, Eq. (11), we write

$$
\int_{\text {total }} E d a+F_{w}=0
$$

where $E$ is the net evaporation minus precipitation. Note that the integral in (26) also comprises precipitation over land, which through river runoff influences the oceanic freshwater budget. To compute $E$ over the ocean, one has to consider the ratio $\epsilon_{w}$ of the ocean area to the catchment area of the ocean basin. Taking $E$ to be constant along a latitude circle, the range of $\epsilon_{w}$ is

$$
\epsilon \leqslant \epsilon_{w} \leqslant 1,
$$

meaning that in one extreme $\left(\epsilon_{w}=1\right)$ the ocean basin receives only the moisture transported in the atmosphere right above it, whereas in the other extreme $\left(\epsilon_{w}\right.$ $=\epsilon$, meaning that the catchment area is the entire latitude circle) it catches all the river runoff as well. With the Atlantic in mind, $\epsilon_{w}$ in the range 0.3 to 0.5 is a reasonable value since the Atlantic (including the Arctic) receives runoff from almost the entire Americas and a large portion of Asia (e.g., Baumgartner and Reichel 1975; Broecker et al. 1990). 
Thus, we obtain for $E$ over the ocean

$$
E=\frac{1}{\epsilon_{w}} \frac{F_{w}}{F_{01}}
$$

and combining (28) with the conversion formula from freshwater fluxes into equivalent surface salinity fluxes, Eq. (6), we obtain

$$
H_{S}=\frac{1}{\epsilon_{w}} \frac{S_{0}}{D} \frac{F_{w}}{F_{01}} .
$$

It has been tacitly assumed that whatever water reaches the high-latitude ocean through atmospheric transports has originated from low latitudes of the same ocean, so the same $\epsilon_{k^{\prime}}$ applies at high and low latitudes. In particular, this assumption eliminates cross-basin atmospheric water vapor transports as proposed, for example, by Broecker et al. (1990) to be crucial for the maintenance of the global thermohaline circulation. The present model is too simple to address this issue, which is therefore sidestepped.

Finally, the parameterization (9) for $F_{w}$ is inserted into (29), which, together with the abbreviation (18) for $T_{2}-T_{1}$ and the definition $\gamma \equiv \tilde{\gamma} / F_{01}$, yields

$$
H_{S}=\frac{1}{\epsilon_{w}} \frac{S_{0}}{D} \gamma T \text {. }
$$

With the above choices for $\tilde{\gamma}$ and $F_{01}, \gamma$ is approximately $1.2 \times 10^{-10} \mathrm{~m} \mathrm{~s}^{-1} \mathrm{~K}^{-1}$, equivalent to 3.8 $\mathrm{mm} \mathrm{yr}^{-1}$ change in net high-latitude precipitation per degree change in meridional temperature contrast (if $\epsilon_{\mathfrak{w}^{\prime}}=1$ ). Note that if $H_{S}$ were obtained from a true linearization of the NSM model, $\gamma$ would be larger by a factor of $\sim 3$, since NSM find their meridional fluxes to be proportional to roughly the third power of the temperature gradient.

Total salt content of the model ocean is constant; introducing

$$
S \equiv S_{2}-S_{1},
$$

the difference between the salt conservation equations, (3) and (4), gives

$$
\dot{S}=\frac{2}{\epsilon_{w}} \frac{S_{0}}{D} \gamma T-2|q| S .
$$

The formulation of the model is now complete.

\section{Equilibrium solutions}

The coupled model has been reduced to two prognostic equations for the meridional temperature and salinity differences. For reference, the model equations to be solved are rewritten as

$$
\begin{aligned}
& \dot{T}=\lambda\left(T_{E}-T\right)-2 k|\alpha T-\beta S| T, \\
& \dot{S}=\frac{2}{\epsilon_{W}} \frac{S_{0}}{D} \gamma T-2 k|\alpha T-\beta S| S .
\end{aligned}
$$

Note that Eq. (34) for the salinity contrast is homogeneous; the meridional salinity gradient is forced by the temperature gradient, which itself is maintained by solar radiation and eroded by longwave radiation and meridional atmospheric transports [implicit in the definitions for $T_{E}$ and $\lambda$, Eqs. (21) and (23), respectively], and by oceanic transports. The coupling of the heat and salt budgets not only through the oceanic flow $q$, but also through the temperature-driven freshwater flux, is the most significant difference between the present model and the one by Huang et al. (1992), who prescribe the freshwater flux as an independent parameter.

The structure of the equilibrium solutions is best displayed graphically in $T-S$ phase space. The algebra shown explicitly will be kept to a minimum; rather, the results will be quoted and made plausible.

Figure 2 shows the $T-S$ phase space between the origin and $\beta S=\alpha T_{E}, \alpha T=\alpha T_{E}$. The choice of parameters is $\alpha=2 \times 10^{-4} \mathrm{~K}^{-1}, \beta=0.8 \times 10^{-3} \mathrm{psu}^{-1}$, $T_{E}=30^{\circ} \mathrm{C}, \gamma=2 \times 10^{-10} \mathrm{~m} \mathrm{~s}^{-1} \mathrm{~K}^{-1}, k=2 \times 10^{-8} \mathrm{~s}^{-1}$, $\epsilon=1 / 6$, and $\epsilon_{w}=0.3$. The main diagonal (dash-dotted line) marks $\beta S=\alpha T$ where the influences of temperature and salinity gradients on density gradients exactly cancel and there is no flow $(q=0)$. To the left of the diagonal, the flow is temperature dominated (thermally direct), with near-surface flow toward high latitudes. The long-dashed and solid curves represent, respectively, the equations $\dot{T}=0$ and $\dot{S}=0$. The two curves intersect at the solution points. The temperature equilibrium curve meets the first diagonal at $\left(\alpha T_{E}, \alpha T_{E}\right)$

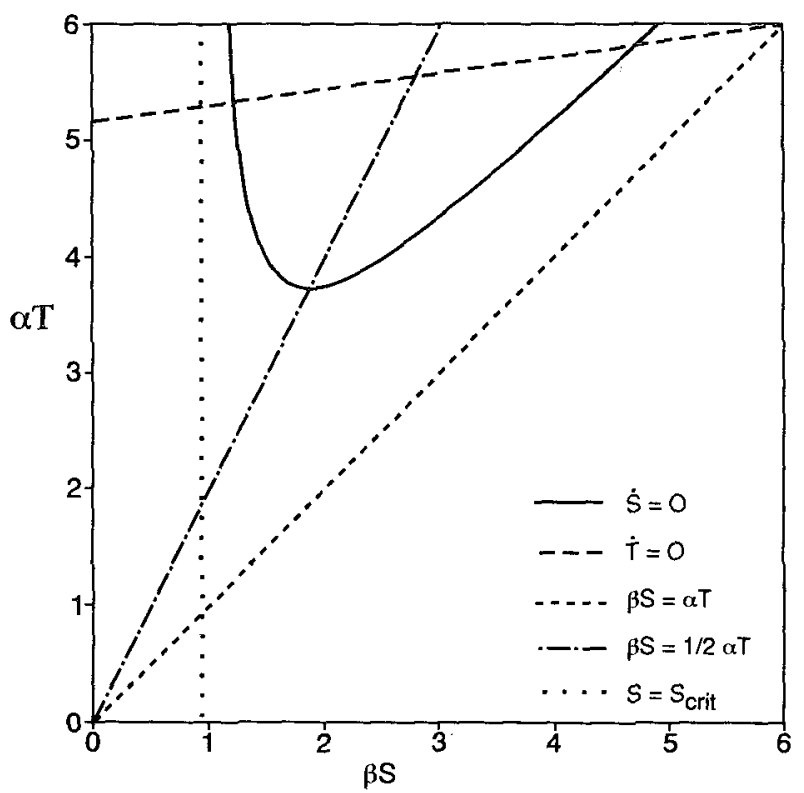

FiG. 2. $T-S$ phase space between the origin and $\left(\beta S=\alpha T_{E}=6\right.$, $\alpha T=\alpha T_{E}=6$ ). Solid curve: $\dot{S}=0$, long-dashed curve: $\dot{T}=0$, dotted line: $S=S_{\text {crit. }}$ Short-dashed: $\beta S=\alpha T$ (no flow, $q=0$ ), and dashdotted: $\beta S=\frac{1}{2} \alpha T$, which intersects the $\dot{S}=0$ curve at its minimum $\left(2 S_{\text {crit }}, 4 S_{\text {crit }}\right)$. 
where the flow vanishes; hence, $T=T_{E}$. The smaller $S$ is the larger $q$ becomes, and $T$ deviates more from $T_{E}$. Since $\lambda \gg q$, for most parameter choices (see section 3 ), the temperature equilibrium curve will hit the $\alpha T$ axis not far away from $T=T_{E}$.

The shape of the $\dot{S}=0$ curve can be deduced qualitatively by considering two limiting cases. First, let $\beta S$ become very large. Then $\alpha T$ must also become large, but such that $|\alpha T-\beta S| \ll \beta S$ because otherwise the linear term on the right side of (34) cannot match the quadratic term. Thus, the salinity equilibrium curve will asymptote a parallel to the diagonal.

The other limiting case is best considered by rearranging the right side of (34), which for $q>0$ can be written as

$$
\dot{S}=-2\left(k \alpha S-\frac{\gamma}{\epsilon_{w^{\prime}}} \frac{S_{0}}{D}\right) T+2 k \beta S^{2}
$$

Thus,

$$
T \rightarrow \infty, \text { for } S \rightarrow S_{\text {crit }} \equiv \frac{1}{k \alpha D} \frac{\gamma}{\epsilon_{w}} S_{0},
$$

and the $\dot{S}=0$ curve must have a minimum at some intermediate point, which is readily shown to be at

$$
\beta S_{\min }=\frac{1}{2} \alpha T_{\min }=2 \beta S_{\text {crit }}
$$

The minimum is where the $\dot{S}=0$ curve meets the dash-dotted line marking $\beta S=\frac{1}{2} \alpha T$.

A physical interpretation is readily given. There are two influences of temperature on the salinity budget, salinity advection by the thermally driven part of the flow and the surface freshwater flux [see Eq. (35)]. For $S>S_{\text {crit }}$, the first term in the brackets of Eq. (35) is larger than the second term. As $S$ gets smaller and approaches $S_{\text {crit }}$, the two influences of temperature on the salinity budget almost cancel, and a very large temperature contrast is required to balance salinity advection by the salinity-driven part of the flow. $S<S_{\text {crit }}$ would mean that $T<0$ in equilibrium, which is nonsensical. We see that salinity conservation alone leads to a lower limit on the equilibrium salinity gradient, which is not the case if the surface freshwater flux is prescribed independent of $T$. In that case, a small $S$ would simply require large $T$ (very strong flow). In either case, the temperature contrast must be supported by the thermal forcing.

Large $S$ requires large $T$ to keep $q$ positive and small, so salinity advection can be balanced by surface freshwater flux despite the large salinity gradients. A large $T$ also causes strong freshwater flux, but its effect on the salinity budget is smaller than the effect of larger temperature-driven salinity advection.

As the $\dot{T}=0$ curve must remain below the $T=T_{E}$ line, Eq. (37) shows as a necessary condition for the existence of two real solutions of (33) and (34) with $q$
$>0$ that the minimum of the $\dot{S}=0$ curve lie below that line; that is,

$$
\frac{1}{k \alpha D} \frac{\gamma}{\epsilon_{w}} \beta S_{0} \equiv \beta S_{\text {crit }}<\frac{1}{4} \alpha T_{E}
$$

For $\alpha=2 \times 10^{-4} \mathrm{~K}^{-1}, \beta=0.8 \times 10^{-3} \mathrm{psu}^{-1}$, and $T_{E}$ $=30^{\circ} \mathrm{C}, S_{\text {crit }}$ must be below 2 psu to make a thermally dominated solution possible. With $\alpha, \beta, D$, and $S_{0}$ given, $S_{\text {crit }}$ is essentially determined by $\gamma /\left(k \epsilon_{w}\right)$, which is an approximate measure of the relative strengths of the atmospheric hydrological cycle and the thermohaline circulation, for a given meridional temperature contrast. Figure 3 shows $S_{\text {crit }}$ and temperature and salinity equilibrium curves for $\gamma=10^{-10} \mathrm{~m} \mathrm{~s}^{-1} \mathrm{~K}^{-1}, \epsilon$ $=1 / 6, \epsilon_{w}=0.3$, and several choices of $k$, beginning with the one exactly obeying the criterion (38). Then, $k$ is successively multiplied by a factor of 1.2 , giving a range of a factor of 2.5 . With growing $k, S_{\text {crit }}$ decreases, and the $\dot{S}=0$ curves move down. The $\dot{T}=0$ curves likewise move down, because the circulation is more efficient in smoothing out temperature contrasts induced by the surface forcing. But the salinity curve moves down with increasing $k$ much faster than the temperature curve, so an equilibrium with poleward surface flow can be enforced in this model through large enough $k$. The resulting salinity gradient, however, may be very small, while the steady-state temperature contrast is not so sensitive to the choice of $k$.

Note that Eqs. (33) and (34) also admit a solution with $q<0$, characterized by $\alpha T<\beta S$ and equatorward surface flow. As this solution always exists and is very similar to the salinity-dominated solutions of other box models, it is not further discussed here. Note also that, in steady state, Eqs. (33) and (34) can be combined into a fourth-order equation in $T$ alone, for both pos-

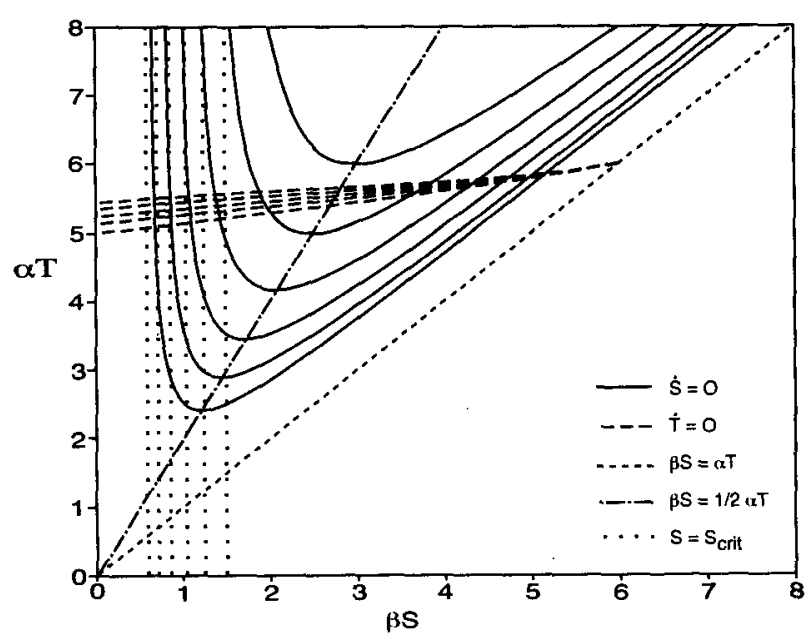

FIG. 3. As in Fig. 2 but between the origin and $\beta S=4 / 3 \alpha T_{E}, \alpha T$. $=4 / 3 \alpha T_{E}$, and $k$ varying by increments of 1.2 . The $\dot{S}=0$ and the $\dot{T}$ $=0$ curves move down as $k$ increases; the $S=S_{\text {crit }}$ line moves to the left. 
itive and negative $q$. For $q>0$, there are two solutions at the intersections of the temperature equilibrium curve with the branch of the salinity equilibrium curve that lies to the left of the pole at $S=S_{\text {crit }}$. These solutions are physically irrelevant and not further discussed here.

\section{Stability analysis}

\section{a. Qualitative discussion}

Figure $4 \mathrm{a}$ is a cutout of Fig. 2 and shows, as vectors, the tendencies in $\alpha T$ and $\beta S$ that are invoked if a point in phase space is not in equilibrium. The $S$ components of the tendencies are very small, illustrating that the temperature gradient equilibrates much faster than the salinity gradient. Oceanic heat conservation, Eq. (33), shows that for a given $T$, greater than steady-state $S$ leads to temperature increase, while anomalously low $S$ leads to temperature decrease. In the range $\alpha T>\beta S$, $S$ larger than its steady-state value always means the point is below the temperature equilibrium; as $T$ goes up, the $T$ curve is approached again.

For the salinity equilibrium curve, Eq. (34), we find that with $S$ given, $S$ decreases if a point lies above the curve, and increases if the point is below the curve. The salinity tendency is thus away from the right-hand branch of the $\dot{S}=0$ curve, which marks instability, and toward the left-hand branch, which is the stable one. Stable, steady solutions with $q>0$ are therefore confined to the interval $S_{\text {crit }}<S<2 S_{\text {crit }}$. These considerations are illustrated by Fig. $4 \mathrm{~b}$, which is the same cutout as Fig. 4a, but only the $\beta S$ component of the tendencies is plotted. Notice that there are strong tendencies to the right across the $q=0$ line, and the saline dominated solution, though not plotted, is identified by the converging arrows in the $q<0$ region.

To evaluate $S_{\text {crit }}$ from Eq. (36), we recall from earlier sections that $\gamma \approx 2 \times 10^{-10} \mathrm{~m} \mathrm{~s}^{-1} \mathrm{~K}^{-1}, D=5000 \mathrm{~m}$, $S_{0}=35 \mathrm{psu}$, and $\epsilon_{w}$ is between $1 / 6$ and 1 . A value for the product $k \alpha$ can be estimated by noting that a temperature contrast of $29^{\circ} \mathrm{C}$ leads to an overturning with a flushing timescale $q^{-1}$ of $10^{10} \mathrm{~s}$ (ignoring, for the sake of argument, the braking action of the salinity gradient). This gives $k \alpha \approx 4 \times 10^{-12} \mathrm{~s}^{-1} \mathrm{~K}^{-1}$, and

$$
S_{\text {crit }}=\frac{1}{k \alpha D} \frac{\gamma}{\epsilon_{w}} S_{0} \approx \frac{1}{\epsilon_{w}} 0.35 \text { psu. }
$$

The lower bound on the steady-state meridional salinity contrast, $S_{\text {crit }}$, ranges between roughly 0.4 and 2 psu; the corresponding upper bound on stable $S$ is $2 S_{\text {crit }}$, which lies between 0.8 and 4 psu. Evaluation of (38) shows that if the freshwater flux forcing is so strong that $S_{\text {crit }}$ approaches $2 \mathrm{psu}$, the salinity equilibrium curve may hardly dip below the $T=T_{E}$ line in the $T$ $S$ diagram, making the stable branch very small. The possible stable solutions are then very vulnerable to small, finite perturbations.

If a third-power law is assumed for the atmospheric moisture transport instead of the linear relationship, Eq. (9), the topology of the curves on which $\dot{S}=0$ changes considerably; in particular, $T$ is then a multivalued function of $S$ on the $\dot{S}=0$ curve. The solutions

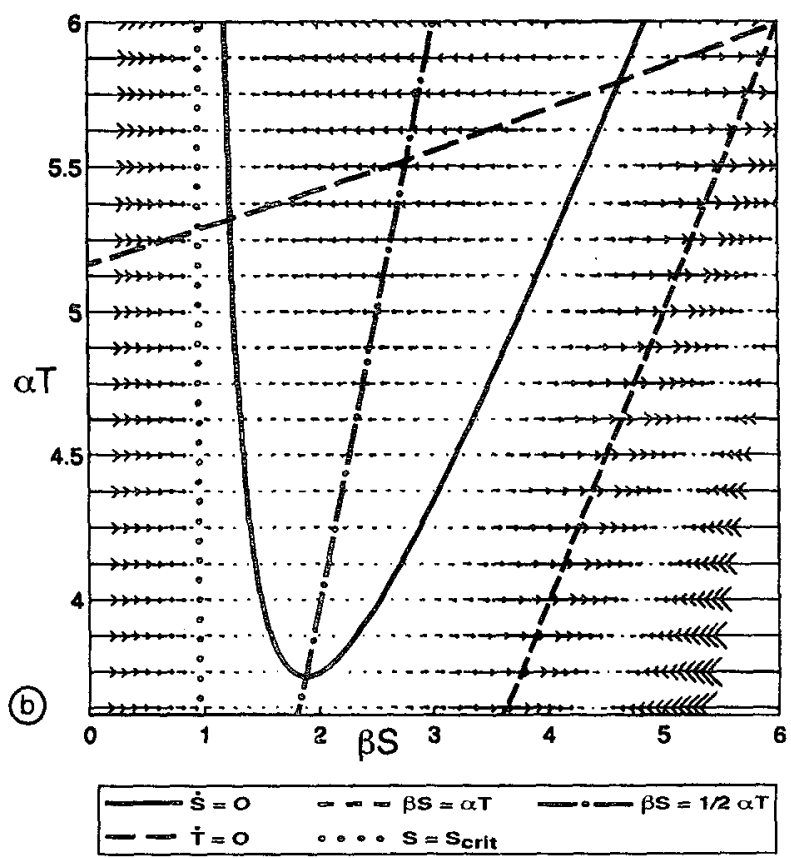

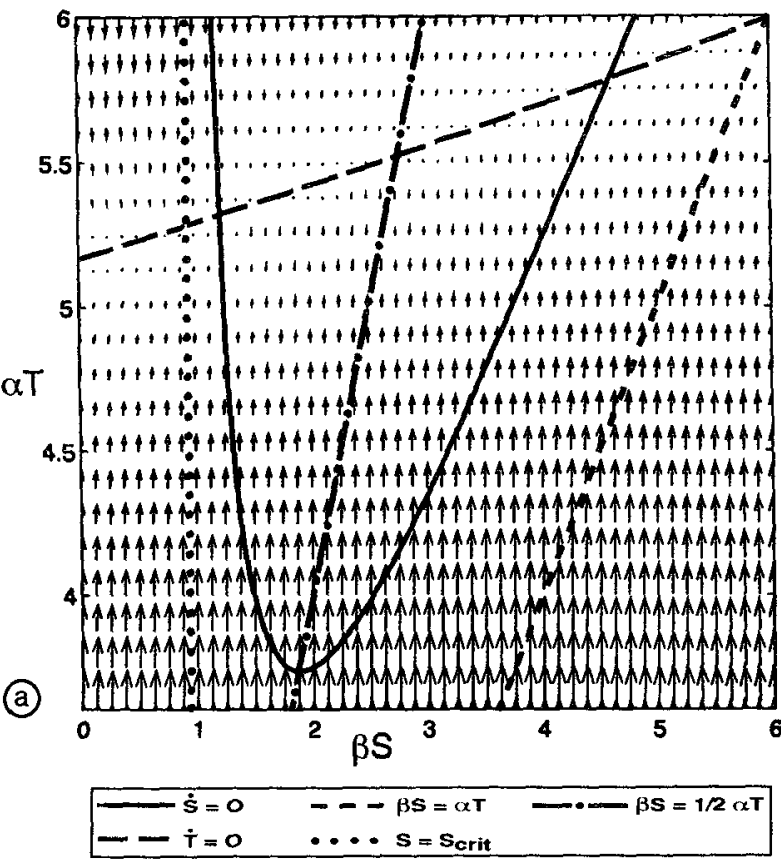

FIG. 4. (a) Detail of Fig. 2 with the tendencies added as arrows. (b) As in (a) but only for the salinity tendencies. 
do not change significantly, however; in particular, there are still two equilibria with $q>0$, and the line $\beta S=\frac{1}{2} \alpha T$ still separates the stable from the unstable solutions. We are thus confident that our stability analysis is robust to details of the representation of atmospheric transports.

\section{b. Linear perturbation analysis}

To analyze the stability of the high-latitude sinking equilibrium $(q>0)$ to infinitesimal perturbations, we write

$$
T=\bar{T}+T^{\prime}, \quad S=\bar{S}+S^{\prime}
$$

in the heat and salt conservation equations, (33) and (34); the overbar marks an equilibrium value. One obtains, after keeping terms only to first order,

$$
\begin{aligned}
& \dot{T}^{\prime}=-[\lambda+2 k(2 \alpha \bar{T}-\beta \bar{S})] T^{\prime}+2 k \beta \bar{T} S^{\prime} \\
& \dot{S}^{\prime}=2\left(\frac{\gamma S_{0}}{\epsilon_{n} . D}-k \alpha \bar{S}\right) T^{\prime}-2 k(\alpha \bar{T}-2 \beta \bar{S}) S^{\prime} .
\end{aligned}
$$

Equations (41) and (42) can be rewritten as

$$
\left(\begin{array}{c}
\alpha \dot{T}^{\prime} \\
\beta \dot{S}^{\prime}
\end{array}\right)=\mathbf{A}\left(\begin{array}{c}
\alpha T^{\prime} \\
\beta S^{\prime}
\end{array}\right)
$$

where

$$
\mathbf{A} \equiv\left(\begin{array}{ll}
a & c \\
d & b
\end{array}\right)
$$

with

$$
\begin{aligned}
a & \equiv-[\lambda+2 k(2 \alpha \bar{T}-\beta \bar{S})]<0, \\
b & \equiv-2 k(\alpha \bar{T}-2 \beta \bar{S}) \quad \text { nondefinite, } \\
c & \equiv 2 k \alpha \bar{T}>0 \\
d & \equiv 2\left(\frac{\gamma \beta S_{0}}{\epsilon_{u} \cdot \alpha D}-k \beta \bar{S}\right) \quad \text { nondefinite. }
\end{aligned}
$$

Note that in principle $d$ is nondefinite, but the requirement that steady-state salinity be larger than $S_{\text {crit }}$ renders $d$ negative [see Eq. (36)]. Using the abbreviation

$$
\bar{q} \equiv k(\alpha \bar{T}-\beta \bar{S})
$$

Eqs. (45a,b) can be rewritten as

$$
\begin{aligned}
& a \equiv-\lambda-2 \bar{q}-2 k \alpha \bar{T}, \\
& b \equiv-2 \bar{q}+2 k \beta \bar{S} .
\end{aligned}
$$

All feedbacks present in this model can be identified from Eq. (45), in conjunction with (43) and (44). The matrix element $a$ shows that if $T^{\prime}$ is positive (say), it is reduced by the Newtonian cooling law, by increased advection through the mean flow as the temperature gradient has increased and by increased advection of mean temperature gradient through anomalous flow. From $b$ we see that if $S^{\prime}>0$, increased advection by the mean flow reduces the anomaly, whereas the reduction in flow strength causes reduced advection of mean salinity gradient, further increasing $S^{\prime}$. By reduction of flow strength, $S^{\prime}>0$ also leads to increases in $T^{\prime}(c>0)$. A positive $T^{\prime}$ (caused possibly by positive $S^{\prime}$ ) causes two competing effects on the salinity gradient, enhancement through a more vigorous atmospheric moisture flux and reduction through a stronger oceanic flow and hence advection of mean salinity gradient. The first effect constitutes a positive feedback between the thermohaline circulation and the atmospheric moisture flux, which has been the focus of NSM and was dubbed eddy moisture transport-thermohaline circulation (EMT) feedback.

In the box model of Marotzke (1990), $T$ was kept fixed, as was the surface freshwater flux. The only feedbacks present are therefore the ones encompassed in element $b$ of $\mathbf{A}$ and $b$ greater or smaller than zero (equivalent to $\beta S$ greater or smaller than $\frac{1}{2} \alpha T$ ), determines whether the steady state is unstable or stable. In the present coupled model, the same criterion still divides the salinity equilibrium curve (Fig. 2) into a stable branch and an unstable branch, because the flow strength depends linearly on density gradient. Huang et al. (1992) admit temperature variations, which however have no influence on the surface freshwater flux; this can be interpreted as setting $\gamma$ to zero in (45d).

To analyze how all the feedbacks act together, the eigenvalues of $\mathbf{A}$ must be computed, which are readily found to be

$$
\mu_{1 / 2}=\frac{1}{2}(a+b) \pm\left[\left(\frac{a+b}{2}\right)^{2}-(a b-c d)\right]^{1 / 2} .
$$

As

$$
a+b=-\lambda-6 \bar{q}<0,
$$

one eigenvalue of $\mathbf{A}$ is always negative, whereas the other is positive if

$$
\operatorname{det}(\mathbf{A})=\mu_{1} \mu_{2}=a b-c d<0 .
$$

If both $a$ and $b$ are negative, there can still be a growing mode if $c$ and $d$ have the same sign and are large enough. Inspection of (45) shows that without an interactive moisture flux, $d<0$ and $c d<0$ always. Nonzero $\gamma$, however, increases $d$, and even though in this simple model it cannot make $d$ positive and thus overthrow the criterion (49), the interactive moisture flux will play a role in the case of finite-amplitude perturbations, as demonstrated by NSM.

Using the definitions of $a, b, c$, and $d$, Eq. (45), we obtain for $\operatorname{det}(\mathbf{A})$ in a mixed notation

$$
\operatorname{det}(\mathbf{A})=-\lambda b+8 \bar{q}^{2}-4 k \bar{T} \frac{\gamma}{\epsilon_{w} D} \beta S_{0} .
$$

Positive contributions to $\operatorname{det}(\mathbf{A})$ stabilize the thermohaline circulation, while negative contributions destabilize it. We see that the mean circulation stabilizes 
since it reduces anomalous temperature and salinity gradients. The atmospheric moisture flux always destabilizes, through the EMT feedback. A strong Newtonian cooling of ocean (surface) temperature, characterized by large $\lambda$, is stabilizing if $b$, standing for the purely oceanic interaction between salinity contrast and the thermohaline circulation, is negative (characterizing a damped mode). Strong restoring of $T$ toward $T_{E}$ prevents the large increase of $T$ owing to weakened overturning caused by increased atmospheric moisture flux. If, in contrast, $b$ is positive, characterizing the positive feedback that has led to transitions in models using mixed boundary conditions, a large $\lambda$ will amplify this positive feedback. In particular, if a state is marginally stable, as has often happened in GCM studies (e.g., Marotzke 1990; Zhang et al. 1993), a weaker Newtonian cooling of SST (while still $\lambda \gg|q|$ ) may prevent the otherwise ensuing collapse of the thermohaline circulation, as shown by Zhang et al. (1993). The reason is that unless SST is restored very quickly, an initially weakened thermohaline circulation leads to lower high-latitude temperatures, which invigorates convective sinking, thus establishing a negative feedback.

Note, however, that stable states are made more stable by strong Newtonian cooling if the EMT feedback is dominant. Note also that in the present interpretation it has been assumed, effectively, that $\lambda$ and $b$ can be varied leaving $\bar{q}$ and $\bar{T}$ constant. However, a more thorough analysis of the eigenvalue dependence on the model parameters shows that the variations in $\bar{q}$ and $\bar{T}$ are minor and do not change the qualitative conclusions given above.

\section{Effects of "flux adjustment" on the stability behavior}

\section{a. Conventional flux adjustment}

Coupled ocean-atmosphere GCMs have large difficulties representing current climate. For example, an atmospheric GCM and an oceanic GCM may be spun up separately and produce today's climate reasonably well. Upon coupling, however, the models often drift into a quite different climate state (e.g., Sausen et al. 1988 ). The climate drift has been associated with incompatible surface fluxes of the oceanic and atmospheric spinup, respectively. As a remedy, some or all the surface fluxes are "adjusted" (i.e., artificial sources introduced at the sea surface) by an amount that, once it is specified, is independent of subsequent changes in the model state. Flux adjustments have been applied in the most recent published $\mathrm{CO}_{2}$ increase studies $(\mathrm{Cu}-$ basch et al. 1992; Manabe and Stouffer 1993). Because the flux adjustments are fixed in time (or follow a fixed seasonal cycle), it has been argued that they do not affect the dynamical behavior of linear models (Sausen et al. 1988).
While the introduction of artificial sources of, say, heat and freshwater at the sea surface has been widely met with criticism (and sometimes ridicule), because it casts doubt upon the reliability of results obtained that way, little has been done to explore the effects of flux adjustments in a systematic way. The simple coupled model discussed here allows exactly this: we follow the procedure of NSM who used the fully nonlinear, numerical counterpart of the present model.

Flux adjustments are used because of incompatible fluxes of the oceanic and atmospheric components, which arise because of errors somewhere in the physics of either or both subsystems. In our model the physics consists of the radiative shortwave and longwave fluxes and three formally independent horizontal transports. The radiative fluxes enter through the constants $A_{2}-A_{1}$ and $B$ in Eq. (19). Here $A_{2}-A_{1}$ represents the differential shortwave heating at the top of the atmosphere. In our model there is no feedback associated with this term, and therefore an error in $A_{2}-A_{1}$ would be corrected perfectly by a fixed additive adjustment to the surface heat flux (note, however, that this would not be true in a model with albedo-temperature feedback). The constant $B$ represents the feedback between longwave emissions and surface temperature, and it only enters as an addition to the dynamical feedback coefficient $2 \chi$ [see Eq. (19)]. Thus, errors in $B$ can be corrected by any prescription that works for correcting errors in $\chi$. Consequently, we only need to consider errors in the three horizontal transports, $H_{d}$ and $F_{w}$ in the atmosphere and $q$ in the ocean, given by (8), (9), and (5), respectively. Atmospheric transports will be discussed first.

If either of $H_{d}$ and $F_{w}$ were in error by a simple bias, and thus the surface heat or freshwater flux wrong by a fixed amount, then an additive correction by this amount would yield the correct results for all model variables except for the meridional transport that was in error to the beginning. But it is possible that $H_{d}$ or $F_{w}$ is in error because the respective coefficient, $\tilde{\chi}$ or $\tilde{\gamma}$, is wrong-implying some incorrect dynamics, numerics, or parameterization.

Assume, first, that $\tilde{\gamma}$ is too large, leading to an overestimation of surface freshwater flux with too strong net precipitation at high latitudes. Even though the steady-state value may be adjusted, giving the correct steady-state salinity gradient, any perturbation will cause the erroneous $\tilde{\gamma}$ to become active again. To see the consequences, analyze $\operatorname{det}(\mathbf{A})$, Eq. (50). Overestimation of $\tilde{\gamma}$ and thus $\gamma$ makes $\operatorname{det}(\mathbf{A})$ more negative than it should be, erroneously destabilizing the steady state (potentially, one eigenvalue could be rendered positive that otherwise would be negative). Too weak $\tilde{\gamma}$ (underestimated atmospheric hydrological cycle) would lead to an underestimation of the sensitivity of the steady state.

Too large $\tilde{\chi}$ means an overestimation of atmospheric heat transport, and thus of the strength of the coeffi- 
cient, $\lambda$, controlling Newtonian cooling of ocean temperature. The effects of varying $\lambda$ have been discussed in the previous section and apply directly. Overestimating $\lambda$ leads to artificially large sensitivity if the purely oceanic feedback between salinity contrasts and the thermohaline circulation is induced (possibly by finite-amplitude perturbations) and to artificially small sensitivity if the positive feedback between atmospheric moisture flux and the thermohaline circulation dominates.

NSM have performed experiments with their nonlinear model in which they have increased or decreased meridional atmospheric transports by a fixed percentage (as assumed here), the incurred error was corrected so as to obtain the steady state defined as the standard, which was then subjected to finite-amplitude perturbations. NSM determined the threshold values for negative high-latitude salinity anomalies that induce a transition to a low-latitude equilibrium and have indeed found that underestimating atmospheric heat transport, by itself, led to an underestimation of the stability of the high-latitude sinking state. If both atmospheric heat and moisture fluxes are underestimated, however, the steady state is slightly more stable than if only moisture fluxes were underestimatedunderestimating atmospheric heat transports now stabilizes. This behavior is consistent with the discussion of the present model: if atmospheric moisture flux is artificially weakened, the EMT feedback does not become important after the perturbation is applied, and weaker atmospheric heat transport, leading to weaker Newtonian cooling, stabilizes the thermohaline circulation. If atmospheric moisture transport has full strength, too strong atmospheric heat transport erroneously prevents the EMT feedback from increasing the meridional temperature gradient beyond the finiteamplitude instability threshold.

We conclude that flux adjustments may help attain the correct mean climate, but they do not improve the erroneous time-dependent model behavior caused by incorrect atmospheric transports.

Incompatibilities between oceanic and atmospheric transports may also arise because the oceanic transports of mass and heat are in error. Underestimating the meridional overturning circulation $q$ makes the steady state more unstable, according to Eq. (50), which is plausible since a strong circulation is likely to be more stable than a weak one. In particular, underestimation of $q$ might occur because the factor, $k$, relating $q$ to the meridional density contrast [see Eq. (5)], might be too small. Figure 3 shows that for small $k$, the steady states are more unstable to finite-amplitude salinity perturbations than for large $k$. As a rule of thumb, the model will turn into a low-latitude sinking equilibrium if it is placed to the right of the right-hand branch of the salinity equilibrium curve. Consequently, it is important to model the strength of the thermohaline cir- culation realistically if the correct stability behavior is to be determined.

\section{b. Alternate flux adjustment schemes}

The previous section shows that the correct dynamics, that is, the correct relation between the climate "state" ( $T$ and $S$ ) and the transports, is essential for obtaining the correct sensitivity of our simple model. The dynamics is encapsulated in the parameters $2 x$ $+B, \gamma$, and $k$. We have shown that if these parameters are in error, so is the transient behavior of the model, even though the correct mean state may have been attained by adding flux adjustments at the sea surface. We will show now that the correct stability behavior in our model can be recaptured if the conventional additive adjustment scheme is replaced by appropriate alternate schemes. Which scheme is appropriate depends on where the error is located. We will thus derive the correct remedy assuming different sources of error.

\section{1) ERROR IN ATMOSPHERIC MOISTURE TRANSPORT}

Let us first assume that the only wrong coefficient is the constant linking meridional temperature gradient and the atmospheric moisture flux, that is, $\gamma$. An incorrect moisture flux causes erroneous salinity gradients and hence oceanic circulation strength and temperature gradients. For positive $q$, the steady-state version of the equation for the salinity gradient, Eq. (34), then reads

$$
\frac{2}{\epsilon_{w}} \frac{S_{0}}{D} \gamma_{F} \bar{T}_{F}-2 \bar{q}_{F} \bar{S}_{F}=0,
$$

where the subscript $F$ stands for "false." Let us assume that we know what the correct $\bar{S}$ is, either from observations or because we have defined a particular set of model parameters as the "truth." By adding to the surface salinity flux the amount, $\Delta H_{S}$, that produces the correct salinity gradient, we obtain the steady-state balance (note that both $k$ and the atmospheric heat budget are assumed correct for the moment):

$$
\frac{2}{\epsilon_{w}} \frac{S_{0}}{D} \gamma_{F} \bar{T}-2 \bar{q} \bar{S}+\Delta H_{S}=0 .
$$

In a numerical model, the surface salinity gradient would be set to $\bar{S}$ at every timestep, and $\Delta H_{S}$ diagnosed once a steady state is reached. If we define

$$
H_{S, F}(T) \equiv \frac{2}{\epsilon_{w}} \frac{S_{0}}{D} \gamma_{F} T
$$

for the wrong surface salinity flux caused by an arbitrary temperature gradient and

$$
\bar{H}_{S} \equiv H_{S, F}(\bar{T})+\Delta H_{S}=2 \bar{q} \bar{S}
$$


for the correct surface salinity flux at the correct equilibrium, the traditional flux adjustment would replace $H_{S, F}(T)$ by

$$
H_{S}^{\text {trad }} \equiv H_{S, F}(T)+\Delta H_{S} .
$$

In contrast, we consider adjusting the surface salinity flux as

$$
H_{S}^{\text {adj }} \equiv H_{S, F}(T) \frac{H_{S, F}(\bar{T})+\Delta H_{S}}{H_{S, F}(\bar{T})} .
$$

At the correct steady state, the two equations yield the same result, but if Eq. (55) is used, the erroneous $\gamma_{F}$ becomes active again upon departure from steady state. By using the multiplicative adjustment, Eq. (56), however, we obtain the correct effective $\gamma$ since

$$
\frac{H_{S, F}(\bar{T})+\Delta H_{S}}{H_{S, F}(\bar{T})} \equiv \frac{\bar{H}_{S}}{H_{S, F}(\bar{T})}=\frac{\gamma}{\gamma_{F}}
$$

and, hence,

$$
H_{S}^{\mathrm{adj}}=H_{S, F}(T) \frac{\gamma}{\gamma_{F}}=\frac{2}{\epsilon_{w}} \frac{S_{0}}{D} \gamma T,
$$

which is correct for arbitrary $T$.

\section{2) ERROR IN OCEANIC MASS TRANSPORT}

We assume now that the atmospheric transports of heat and moisture are correct $\left(\lambda, T_{E}\right.$, and $\gamma$ are correct), but that $k$ is in error. Equations (33) and (34) for the temperature and salinity difference then read, in steady state,

$$
\begin{gathered}
\lambda\left(T_{E}-\bar{T}_{F}\right)=2 \bar{q}_{F} \bar{T}_{F}=2 k_{F}\left(\alpha \bar{T}_{F}-\beta \bar{S}_{F}\right) \bar{T}_{F} \\
\frac{2}{\epsilon_{w}} \frac{S_{0}}{D} \gamma \bar{T}_{F}=2 \bar{q}_{F} \bar{S}_{F}=2 k_{F}\left(\alpha \bar{T}_{F}-\beta \bar{S}_{F}\right) \bar{S}_{F} .
\end{gathered}
$$

Since $q \ll \lambda$, we can always write approximately [see Eq. (25)]

$$
\begin{aligned}
\bar{T} \approx\left(1-2 \frac{q(\bar{T})}{\lambda}\right) & T_{E} \approx\left(1-2 \frac{q\left(T_{E}\right)}{\lambda}\right) T_{E} \\
& =T_{E}-2 T_{E} \frac{k}{\lambda}\left(\alpha T_{E}-\beta \bar{S}\right)
\end{aligned}
$$

and

$$
\bar{S} \approx \frac{S_{0}}{\epsilon_{w} D} \frac{\gamma \bar{T}}{q\left(T_{E}\right)} \approx \frac{S_{0}}{\epsilon_{w} D} \frac{1}{\left(\alpha T_{E}-\beta \bar{S}\right)} \frac{\gamma}{k} \bar{T} .
$$

We see that the salinity gradient is affected by an erroneous $k$ much more strongly than is the temperature gradient because the latter is strongly tied to $T_{E}$. If the temperature and salinity gradients are forced to take the correct values, $\bar{T}$ and $\bar{S}$, by adding the required fluxes at the surface, the steady-state equations become

$$
\begin{aligned}
& \lambda\left(T_{E}-\bar{T}\right)+\Delta H_{T}=2 k_{F}(\alpha \bar{T}-\beta \bar{S}) \bar{T} \\
& \frac{2}{\epsilon_{w}} \frac{S_{0}}{D} \gamma \bar{T}+\Delta H_{S}=2 k_{F}(\alpha \bar{T}-\beta \bar{S}) \bar{S}
\end{aligned}
$$

or

$$
\begin{aligned}
\bar{H}_{T} & \equiv \lambda\left(T_{E}-\bar{T}\right)=2 k_{F}(\alpha \bar{T}-\beta \bar{S}) \bar{T}-\Delta H_{T} \\
\bar{H}_{S} & \equiv \frac{2}{\epsilon_{w}} \frac{S_{0}}{D} \gamma \bar{T}=2 k_{F}(\alpha \bar{T}-\beta \bar{S}) \bar{S}-\Delta H_{S},
\end{aligned}
$$

where $\vec{H}_{T}$ and $\bar{H}_{S}$ are the correct surface fluxes at the correct climate state. The best interpretation of Eqs. (65) and (66) is that the incorrect oceanic heat and salt transport divergences are augmented by the artificial fluxes, $-\Delta H_{T}$ and $-\Delta H_{S}$, to match the correct atmospheric transport divergences, $\bar{H}_{T}$ and $\bar{H}_{S}$. Traditional flux adjustment would add $\Delta H_{T}$ and $\Delta H_{S}$ to the model-computed surface fluxes at each time step. In contrast, we consider adjusting the surface fluxes according to

$$
\begin{gathered}
H_{T}^{\mathrm{adj}} \equiv H_{T}(T) \frac{\bar{H}_{T}+\Delta H_{T}}{\bar{H}_{T}} \\
H_{S}^{\mathrm{adj}} \equiv H_{S}(T) \frac{\bar{H}_{S}+\Delta H_{S}}{\bar{H}_{S}} .
\end{gathered}
$$

In the model considered here, we have for the case of correctly computed atmospheric fluxes, at the correct mean climate state,

$$
\begin{gathered}
\lambda\left(T_{E}-\bar{T}\right)=2 \bar{q} \bar{T}=2 k(\alpha \bar{T}-\beta \bar{S}) \bar{T} \\
\frac{2}{\epsilon_{w}} \frac{S_{0}}{D} \gamma \bar{T}=2 \bar{q} \bar{S}=2 k(\alpha \bar{T}-\beta \bar{S}) \bar{S}
\end{gathered}
$$

Upon combining Eqs. (63), (64), and (67)-(70), the adjusted fluxes are obtained as

$$
\begin{aligned}
& H_{T}^{\mathrm{adj}}=H_{T}(T) \frac{k_{F}}{k}=\lambda \frac{k_{F}}{k}\left(T_{E}-T\right) \\
& H_{S}^{\mathrm{adj}}=H_{S}(T) \frac{k_{F}}{k}=\frac{2}{\epsilon_{w}} \frac{S_{0}}{D} \gamma \frac{k_{F}}{k} T .
\end{aligned}
$$

Thus, we have effectively replaced the correct $\lambda$ and $\gamma$ by $\lambda k_{F} / k$ and $\gamma k_{F} / k-a$ drawback rather than progress, it may seem. A look at the linearized perturbation equations (41) and (42), however, reveals that with the proposed flux adjustment scheme the right-hand sides are uniformly distorted by the factor $k_{F} / k$, meaning that if the timescale is stretched by $k / k_{F}$, the correct linear behavior near the correct steady state is captured exactly. It is merely necessary to reinterpret time intervals according to the stretched time.

\section{3) INCORRECT ATMOSPHERIC HEAT BALANCE}

Now we assume that the only error is in the coefficient linking the meridional temperature gradient to 
the atmospheric heat flux, that is, in $\chi$. (As noted above, errors in the longwave radiative coefficient can be treated in precisely the same way.) In this case $\lambda$ and $T_{E}$ are incorrect, but $\lambda T_{E}$ is correct [see Eqs. (21) and (23)]. The steady-state equations for the temperature difference are then, for the cases without and with artificial fluxes, respectively,

$$
\begin{gathered}
H_{T, F}\left(\bar{T}_{F}\right)=\lambda_{F}\left(T_{E, F}-\bar{T}_{F}\right)=2 \bar{q}_{F} \bar{T}_{F} \\
\bar{H}_{T} \equiv H_{T, F}(\bar{T})+\Delta H_{T}=\lambda_{F}\left(T_{E, F}-\bar{T}\right) \\
+\Delta H_{T}=2 \bar{q} \bar{T}=\lambda\left(T_{E}-\bar{T}\right) .
\end{gathered}
$$

Since

$$
\lambda_{F} T_{E, F}=\lambda T_{E},
$$

the appropriate flux adjustment is not analogous to the previous procedures, but rather

$$
H_{T}^{\mathrm{adj}}=H_{T, F}(T)+\frac{T}{\bar{T}} \Delta H_{T}
$$

which can be viewed as a combination of an additive and a multiplicative flux adjustment. Equation (76) reduces to the first equality in Eq. (74) at $T=\bar{T}$; for arbitrary $T$, Eq. (76) yields

$$
\begin{aligned}
H_{T}^{\mathrm{adj}}=\lambda_{F}\left(T_{E, F}-T\right) \\
+\frac{T}{\bar{T}}\left[\lambda\left(T_{E}-\bar{T}\right)-\lambda_{F}\left(T_{E, F}-\bar{T}\right)\right] .
\end{aligned}
$$

It is readily seen that with (75), Eq. (77) collapses to

$$
H_{T}^{\mathrm{adj}}=\lambda\left(T_{E}-T\right),
$$

so the adjusted fluxes are identical to the correct ones.

\section{4) UNKNOWN DISTRIBUTION OF ERRORS}

In practice, it is not known what combination of errors in atmospheric and oceanic transports causes flux incompatibilities. Our discussions above assume that there is a single error and that the source of the error is known. There is of course no problem with single or multiple errors if the errors are small-then the prescriptions discussed above can be linearized, they all reduce to the conventional additive flux correction, and the model's stability characteristics are preserved. However, the flux incompatibilities in all coupled atmosphere-ocean GCMs examined to date are order one (Gates et al. 1993). When this is the case and there are multiple sources of error, it is not obvious that there is any one flux prescription scheme that will work well, or best. One could analyze the performance of the various prescription schemes discussed above in the presence of multiple large errors using a more fully nonlinear model, such as the NSM model, but we will not attempt such an analysis here. Nevertheless, the analysis of our simple model makes it clear that the conventional additive flux adjustment scheme leaves the feedbacks associated with fluxes that have erroneous representations uncorrected. Consequently, coupled ocean-atmosphere models that have flux incompatibilities of order one cannot be expected to represent climate sensitivity accurately.

\section{Discussion}

A simple coupled ocean-atmosphere model has been presented, which allows an analytical analysis of the interaction between zonally integrated atmospheric and oceanic meridional transports. The ocean model is the two-box model of Stommel (1961); the atmospheric model consists likewise of two boxes, and has zero heat and moisture capacities and a linear radiation budget. Meridional atmospheric transports of heat and moisture depend linearly on the meridional temperature gradient, reflecting in the simplest possible way that the atmospheric eddy transports of heat and moisture, which dominate at middle and high latitudes, increase as the meridional temperature gradient increases. The model is most immediately a simplification of the coupled model developed by Nakamura et al. (1994), who employed a similar ocean model but a more sophisticated representation of atmospheric transports, sometimes referred to here as "nonlinear box model."

Most of the model behavior can be expressed with two equations for meridional oceanic temperature and salinity gradients. A Newtonian cooling law is derived, without any additional approximation or assumption, for the differential heating that forces oceanic temperature gradients. The target temperature gradient of the Newtonian law is the temperature gradient the model would attain if there was no oceanic heat transport; it is called the atmospheric equilibrium temperature gradient. The restoring coefficient of the Newtonian law is proportional to the efficiency by which atmospheric heat transports can erase temperature gradients and inversely proportional to the relative ratio of ocean area to total area. This geometric factor can significantly increase the degree to which the atmosphere controls oceanic surface temperature gradients; it enters because land is in energy balance, and the ocean must provide or accept whatever amount of heat is necessary to achieve atmospheric heat balance in an entire latitude band. If one makes the extreme assumption that the Atlantic thermohaline circulation is solely responsible for ocean heat transport, a Newtonian restoring coefficient appropriate for basin-scale gradients in the Atlantic is of the order $25 \mathrm{~W} \mathrm{~m}^{-2} \mathrm{~K}^{-1}$, equivalent to a 3-month restoring timescale for a 50-m deep model surface layer. The effective restoring coefficient triples if atmospheric heat transport depends on the third power of the meridional temperature contrast, which gives a restoring timescale of only 1 month (consistent with NSM). But notice that the restoring timescale would be six times as large on a latitude circle entirely covered by ocean. 
The surface freshwater flux, or equivalent surface salinity flux, is essentially the atmospheric meridional moisture flux divided by the ratio of ocean area to catchment area. This geometric factor accounts for the river runoff.

The coupled box model admits the same set of physically meaningful equilibria as do related uncoupled models (Stommel 1961; Marotzke 1990; Huang et al. 1992 ). However, the linear dependence of the surface freshwater flux on the meridional temperature gradient reduces the area in phase space taken up by stable solutions with high-latitude sinking, compared to models with freshwater flux prescribed as an external parameter. Since the oceanic temperature gradient is always close to the atmospheric equilibrium temperature gradient, for physically reasonable oceanic density gradients and hence flow strengths, the equilibrium solutions depend essentially on the relative strengths of surface freshwater flux forcing and the efficiency of oceanic advection; the latter is expressed here as a coefficient relating density gradient to the strength of the thermohaline circulation.

A linear stability analysis of the high-latitude sinking equilibrium allows a quantitative discussion of the feedbacks present. The model allows the same feedback between the thermohaline circulation and the salinity gradient as described in Marotzke (1990). The feedback is positive if the impact of the salinity gradient on the density gradient is more than half the influence of the temperature gradient; otherwise, the feedback is negative. The feedback between ocean circulation, ocean heat transport, and temperature gradient is always negative. The interaction between the temperature-dependent atmospheric moisture flux and the thermohaline circulation creates a positive feedback, as described by Nakamura et al. (1994), who call it "eddy moisture transport-thermohaline circulation" or EMT feedback.

The most complex feedback behavior arises from interactions between atmospheric heat transport and the thermohaline circulation, which can be stabilizing or destabilizing. If atmospheric heat transports are very efficient in removing anomalous temperature gradients (equivalent to a large restoring coefficient in the Newtonian law), anomalies in oceanic flow strength will only weakly influence the temperature distribution. A weakened thermohaline circulation, caused perhaps by a finite-amplitude, negative salinity perturbation at high latitudes, can then lead to decreased ocean salt transport (enhancing the initial anomaly), without increasing the meridional temperature gradient by much. Consequently, the stabilizing effect of a response in temperature gradient is weak, even though the flow is enhanced somewhat by the larger temperature gradient. Thus a very efficient atmospheric heat transport is destabilizing. Indeed, it has been shown recently (Zhang et al. 1993; Rahmstorf and Willebrand 1995) that using restoring timescales larger than one month, which is equivalent to reducing the efficiency of the atmospheric transports, significantly stabilizes the thermohaline circulation in ocean general circulation models. The reason is, in large part, the stronger negative feedback between surface temperature and the thermohaline circulation.

There is, however, a stabilizing effect associated with very strong Newtonian cooling. If the temperature gradient is tightly confined, so is the atmospheric moisture transport, and the EMT feedback is less powerful. It is thus not clear whether the thermohaline circulation in a coupled ocean-atmosphere model would be more stable or less stable if the mean atmospheric heat transport efficiency were increased.

The insight gained about the various feedbacks between oceanic and atmospheric transports carries over directly to an analysis of the stability behavior of coupled models that have been "flux adjusted" to produce the correct mean climate. Flux adjustments are performed to correct for incompatible transport divergences in model ocean and model atmosphere, which are in turn caused by errors in the atmospheric heat or moisture transports, or oceanic mass and hence heat and salt transports, or any combination of these. If a transport is in error, so is the associated feedback. Too strong atmospheric moisture transport leads to artificial destabilization of the thermohaline circulation, as does too weak oceanic mass transport. The effect of too strong atmospheric heat transport can be stabilizing if the EMT feedback is counteracted and destabilizing if the purely oceanic feedback between the salinity gradient and the thermohaline circulation is considered.

Flux adjustments added to the model-computed surface fluxes in order to correct for erroneous representations of model physics still leave the model with erroneous transient behavior. However, it is possible to restore the model's correct transient behavior with nonadditive flux adjustments if there is only one source of the error and that source is known. To assess fully the virtues and drawbacks of nonadditive flux adjustments, a quantitative analysis with a more sophisticated model than the one employed here is necessary. Such tests could be done with still idealized coupled models in which some transports are distorted in a controlled manner and as pure sensitivity studies in coupled GCMs investigating whether different types of adjustment cause different transient model behavior. Note that in models with higher horizontal resolution than ours, the multiplicative flux adjustment should be applied to the horizontal transports rather than to the surface fluxes, in order to conserve mass and moisture. Also, a multiplicative flux adjustment can only change the magnitude of the surface fluxes but not the pattern, and it is not a substitute for ultimately eliminating the error sources in the GCMs.

Feedbacks affecting the thermohaline circulation are often discussed in what is effectively a "two-box" framework (e.g., Willebrand 1993; Marotzke 1994), 
meaning that only large-scale meridional gradients of temperature and salinity are discussed. Our model allows us to formulate these concepts in analytical terms, which is particularly helpful if competing effects are considered, like the impacts of overestimating atmospheric heat transport. We believe that the interpretation of more complex models is made easier by our theoretical investigation.

Acknowledgments. Thirty percent $(\$ 8,695.99)$ of this research was supported jointly by the Northeast Regional Center of the National Institute for Global Environmental Change and by the Program for Computer Hardware, Applied Mathematics, and Model Physics (both with funding from the U.S. Department of Energy). Financial support was also contributed by the Tokyo Electric Power Company through the TEPCO/ MIT Environmental Research Program.

\section{REFERENCES}

Baumgartner, A., and E. Reichel, 1975: Die Weltwasserbilanz. Oldenbourg Verlag, $179 \mathrm{pp}$.

Bretherton, F. P., 1982: Ocean climate modeling. Progress in Oceanography, Vol. 11, Pergamon, 93-129.

Broecker, W. S., T.-P. Peng, J. Jouzel, and G. Russell, 1990: The magnitude of global freshwater transports of importance to ocean circulation. Clim. Dyn., 4, 73-79.

Bryan, F., 1986: High-latitude salinity effects and interhemispheric thermohaline circulations. Nature, 323, 301-304.

Cubasch, U., K. Hasselmann, H. Höch, E. Maier-Reimer, U. Mikolajewicz, B. D. Santer, and R. Sausen, 1992: Time-dependent greenhouse warming computations with a coupled ocean-atmosphere model. Clim. Dyn., 8, 55-69.

Gates, W. L., U. Cubasch, G. Meehl, J. Mitchell, and R. S. Stouffer, 1993: An intercomparison of selected features of the control climates simulated by coupled ocean-atmosphere general circulation models. World Meteorological Organization Publ. WMO/TD-No. 574, $46 \mathrm{pp}$.

Hall, M. M., and H. L. Bryden, 1982: Direct estimates and mechanisms of ocean heat transport. Deep-Sea Res., 29, 339-359.

Haney, R. L., 1971: Surface thermal boundary condition for ocean circulation models. J. Phys. Oceanogr., 1, 241-248.

Held, I. M., 1978: The vertical scale of an unstable baroclinic wave and its importance for eddy heat flux parameterizations. $J$. Atmos. Sci., 35, 572-576.

Huang, R. X., J. R. Luyten, and H. M. Stommel, 1992: Multiple equilibrium states in combined thermal and saline circulation. J. Phys. Oceanogr., 22, 231-246.

Hughes, T. C. M., and A. J. Weaver, 1994: Multiple equilibria of an asymmetric two-basin model. J. Phys. Oceanogr., 24, 619-637.

Manabe, S., and R. J. Stouffer, 1988: Two stable equilibria of a coupled ocean-atmosphere model. J. Climate, 1, 841-866.
, and - 1993: Century-scale effects of increased atmospheric $\mathrm{CO}_{2}$ on the ocean-atmosphere system. Nature, 364, 215-218, , and - 1994: Multiple century response of a coupled oceanatmosphere model to an increase of atmospheric carbon dioxide. J. Climate, 7, 5-23.

Marotzke, J., 1990: Instabilities and multiple equilibria of the thermohaline circulation. Ph.D. thesis. Ber. Inst. Meeresk. Kiel, Germany, $126 \mathrm{pp}$.

- 1994: Ocean models in climate problems. Ocean Processes in Climate Dynamics: Global and Mediterranean Examples, P. Malanotte-Rizzoli and A. R. Robinson, Eds., Kluwer, 79-109. , and J. Willebrand, 1991: Multiple equilibria of the global thermohaline circulation. J. Phys. Oceanogr., 21, 1372-1385.

Nakamura, M., P. H. Stone, and J. Marotzke, 1994: Destabilization of the thermohaline circulation by atmospheric eddy transports. J. Climate, 7, 1870-1882.

North, G. R., 1975: Theory of energy-balance climate models. $J$. Atmos. Sci., 32, 2033-2043.

Rahmstorf, S., and J. Willebrand, 1995: The role of temperature feedback in stabilizing the thermohaline circulation. J. Phys. Oceanogr., 25, 787-805.

Roemmich, D. H., and C. Wunsch, 1985: Two transatlantic sections: Meridional circulation and heat flux in the subtropical North Atlantic Ocean. Deep-Sea Res., 32, 619-664.

Sausen, R., R. K. Barthels, and K. Hasselmann, 1988: Coupled oceanatmosphere models with flux correction. Clim. Dyn., 2, 154163.

Stommel, H., 1961: Thermohaline convection with two stable regimes of flow. Tellus, 13, 224-230.

Stone, P. H., and D. A. Miller, 1980: Empirical relations between seasonal changes in meridional temperature gradients and meridional fluxes of heat. J. Atmos. Sci., 37, 1708-1721.

ally averaged statistical-dynamical model. Part III: The parameterization of the eddy fluxes of heat and moisture. J. Climate, 3, 726-740.

Wang, W.-C., and P. H. Stone, 1980: Effect of ice-albedo feedback on global sensitivity in a one-dimensional radiative-convective climate model. J. Atmos. Sci., 37, 545-552.

Weaver, A. J., and T. M. C. Hughes, 1992: Stability and variability of the thermohaline circulation and its link to climate. Trends in Physical Oceanography, Research Trends Series, Council of Scientific Research Integration, 1, 15-70, 56 pp.

_ E. S. Sarachik, and J. Marotzke, 1991: Freshwater flux forcing of decadal and interdecadal oceanic variability. Nature, 353, 836-838.

_ J. Marotzke, P. F. Cummins, and E. S. Sarachik, 1993: Stability and variability of the thermohaline circulation. J. Phys. Oceanogr., 23, 39-60.

Willebrand, J., 1993: Forcing the ocean with heat and freshwater fluxes. Energy and Water Cycles in the Climate System, E. Raschke, Ed., Springer Verlag, 215-233.

Zhang, S., R. J, Greatbatch, and C. A. Lin, 1993: A reexamination of the polar halocline catastrophe and implications for coupled ocean-atmosphere modeling. J. Phys. Oceanogr., 23, 287-299. 